\title{
DSP implementation of digital pre-distortion in wireless communication systems
}

\begin{abstract}
This paper presents a Digital Signal Processor (DSP) implementation of Digital Pre-distortion (DPD) targeting the 3rd Generation/4th Generation (3G/4G) wireless networks. The nonlinearity of the Power Amplifier (PA) causes several issues such as Adjacent Channel Interference (ACI), power wastage, high energy consumption, and system inefficiency. These shortcomings result in performance limitation for the wireless communications system which is undesirable for the industry. DPD is chosen as the PA linearization method due to its overall advantages in Adjacent Channel Power Reduction (ACPR), cost, efficiency, and implementation flexibility. The DPD system is developed using the C++ Hardware Programming Language in order to be implemented into the target Digital Signal Processor (DSP), the soft-core Microblaze processor by Xilinx. The developed system in $\mathrm{C}++$ is compared with the conventional Matlab Simulation and a performance difference of $0-10 \mathrm{~dB}$ in ACPR is observed. The C++ DSP Implementation is capable of achieving up to $15 \mathrm{~dB}$ of ACPR for WiMAX signal in $4 \mathrm{G}$ networks and $25 \mathrm{~dB}$ for 2-carrier Wideband Code Division Multiple Access (2C-WCDMA) signals in $3 \mathrm{G}$ networks.
\end{abstract}

Keyword: Power amplifier; PA linearization; Digital pre-distortion; MATLAB; C++; 3G; $4 \mathrm{G}$ 